Supplement of Clim. Past, 14, 1213-1228, 2018

https://doi.org/10.5194/cp-14-1213-2018-supplement

(C) Author(s) 2018. This work is distributed under

the Creative Commons Attribution 4.0 License.

(c) (i)

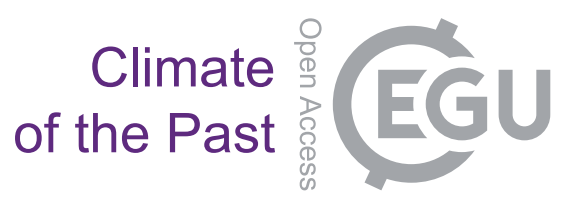

Supplement of

\title{
Response of Pinus sylvestris var. mongolica to water change and drought history reconstruction in the past 260 years, northeast China
}

Liangjun Zhu et al.

Correspondence to: Xiaochun Wang (wangx@ @efu.edu.cn)

The copyright of individual parts of the supplement might differ from the CC BY 4.0 License. 


\section{Supplementary Material}

\section{Supplementary Data}

The following is the regional tree-ring width chronology of Pinus sylvestris in the Central Daxing'an Mountains.

\begin{tabular}{|c|c|c|c|c|c|c|c|c|}
\hline Year & Index & $\begin{array}{l}\text { Sample } \\
\text { depth }\end{array}$ & Year & Index & $\begin{array}{l}\text { Sample } \\
\text { depth }\end{array}$ & Year & Index & $\begin{array}{l}\text { Sample } \\
\text { depth }\end{array}$ \\
\hline 1725 & 1.174 & 1 & 1825 & 0.834 & 67 & 1925 & 0.669 & 108 \\
\hline 1726 & 0.880 & 1 & 1826 & 1.075 & 69 & 1926 & 0.775 & 108 \\
\hline 1727 & 0.637 & 2 & 1827 & 1.058 & 69 & 1927 & 0.826 & 108 \\
\hline 1728 & 1.128 & 2 & 1828 & 1.015 & 71 & 1928 & 0.932 & 108 \\
\hline 1729 & 0.999 & 3 & 1829 & 0.893 & 71 & 1929 & 0.978 & 108 \\
\hline 1730 & 1.159 & 3 & 1830 & 0.994 & 72 & 1930 & 1.048 & 109 \\
\hline 1731 & 0.989 & 3 & 1831 & 0.966 & 73 & 1931 & 0.985 & 109 \\
\hline 1732 & 1.287 & 3 & 1832 & 0.964 & 73 & 1932 & 1.040 & 110 \\
\hline 1733 & 1.062 & 3 & 1833 & 0.993 & 73 & 1933 & 1.130 & 110 \\
\hline 1734 & 0.961 & 3 & 1834 & 0.967 & 73 & 1934 & 1.155 & 110 \\
\hline 1735 & 0.651 & 3 & 1835 & 1.054 & 73 & 1935 & 1.002 & 110 \\
\hline 1736 & 1.121 & 3 & 1836 & 0.841 & 74 & 1936 & 1.023 & 110 \\
\hline 1737 & 1.067 & 5 & 1837 & 0.889 & 74 & 1937 & 0.973 & 110 \\
\hline 1738 & 0.835 & 5 & 1838 & 0.823 & 75 & 1938 & 1.041 & 110 \\
\hline 1739 & 1.052 & 5 & 1839 & 0.800 & 75 & 1939 & 0.902 & 110 \\
\hline 1740 & 1.151 & 5 & 1840 & 1.060 & 77 & 1940 & 0.765 & 110 \\
\hline 1741 & 1.091 & 5 & 1841 & 0.951 & 78 & 1941 & 0.916 & 111 \\
\hline 1742 & 0.596 & 6 & 1842 & 0.921 & 78 & 1942 & 0.998 & 111 \\
\hline 1743 & 0.532 & 6 & 1843 & 0.949 & 78 & 1943 & 1.000 & 113 \\
\hline 1744 & 0.965 & 6 & 1844 & 1.104 & 79 & 1944 & 0.940 & 113 \\
\hline 1745 & 0.775 & 7 & 1845 & 1.163 & 79 & 1945 & 0.812 & 114 \\
\hline 1746 & 1.196 & 7 & 1846 & 1.084 & 79 & 1946 & 0.890 & 114 \\
\hline 1747 & 0.834 & 7 & 1847 & 0.875 & 79 & 1947 & 1.029 & 114 \\
\hline 1748 & 0.509 & 7 & 1848 & 0.870 & 80 & 1948 & 1.170 & 114 \\
\hline 1749 & 0.331 & 7 & 1849 & 0.874 & 81 & 1949 & 1.087 & 114 \\
\hline 1750 & 0.423 & 7 & 1850 & 0.827 & 82 & 1950 & 1.067 & 118 \\
\hline 1751 & 0.756 & 8 & 1851 & 0.660 & 84 & 1951 & 1.071 & 118 \\
\hline 1752 & 0.980 & 8 & 1852 & 0.658 & 84 & 1952 & 1.379 & 118 \\
\hline
\end{tabular}




\begin{tabular}{|c|c|c|c|c|c|c|c|c|}
\hline 1753 & 1.175 & 8 & 1853 & 0.571 & 84 & 1953 & 1.105 & 118 \\
\hline 1754 & 0.975 & 8 & 1854 & 0.707 & 84 & 1954 & 0.933 & 118 \\
\hline 1755 & 1.302 & 8 & 1855 & 0.848 & 84 & 1955 & 0.976 & 118 \\
\hline 1756 & 1.214 & 8 & 1856 & 0.726 & 85 & 1956 & 1.104 & 118 \\
\hline 1757 & 1.180 & 8 & 1857 & 0.881 & 85 & 1957 & 1.009 & 118 \\
\hline 1758 & 1.176 & 8 & 1858 & 0.885 & 85 & 1958 & 1.191 & 118 \\
\hline 1759 & 0.893 & 8 & 1859 & 0.805 & 85 & 1959 & 1.116 & 118 \\
\hline 1760 & 1.054 & 8 & 1860 & 0.663 & 87 & 1960 & 1.048 & 118 \\
\hline 1761 & 1.054 & 8 & 1861 & 0.721 & 88 & 1961 & 0.936 & 118 \\
\hline 1762 & 1.226 & 9 & 1862 & 0.617 & 88 & 1962 & 1.143 & 118 \\
\hline 1763 & 1.243 & 10 & 1863 & 0.618 & 90 & 1963 & 1.047 & 118 \\
\hline 1764 & 1.258 & 10 & 1864 & 0.725 & 91 & 1964 & 1.058 & 118 \\
\hline 1765 & 1.248 & 10 & 1865 & 0.720 & 91 & 1965 & 0.974 & 118 \\
\hline 1766 & 1.334 & 10 & 1866 & 0.751 & 91 & 1966 & 1.006 & 118 \\
\hline 1767 & 1.216 & 10 & 1867 & 1.052 & 91 & 1967 & 0.667 & 118 \\
\hline 1768 & 1.158 & 10 & 1868 & 1.196 & 91 & 1968 & 0.988 & 118 \\
\hline 1769 & 1.276 & 12 & 1869 & 1.175 & 91 & 1969 & 1.018 & 118 \\
\hline 1770 & 1.368 & 14 & 1870 & 1.158 & 91 & 1970 & 0.926 & 118 \\
\hline 1771 & 1.154 & 15 & 1871 & 0.974 & 91 & 1971 & 1.011 & 118 \\
\hline 1772 & 0.816 & 16 & 1872 & 1.092 & 91 & 1972 & 1.072 & 118 \\
\hline 1773 & 0.978 & 17 & 1873 & 1.022 & 91 & 1973 & 1.066 & 118 \\
\hline 1774 & 0.811 & 20 & 1874 & 1.142 & 91 & 1974 & 0.903 & 118 \\
\hline 1775 & 0.965 & 24 & 1875 & 0.821 & 91 & 1975 & 0.959 & 118 \\
\hline 1776 & 0.966 & 25 & 1876 & 1.005 & 91 & 1976 & 1.185 & 118 \\
\hline 1777 & 0.881 & 25 & 1877 & 1.096 & 91 & 1977 & 1.087 & 118 \\
\hline 1778 & 0.976 & 26 & 1878 & 1.208 & 92 & 1978 & 1.145 & 118 \\
\hline 1779 & 1.175 & 28 & 1879 & 0.911 & 93 & 1979 & 0.805 & 118 \\
\hline 1780 & 1.244 & 32 & 1880 & 0.973 & 93 & 1980 & 1.018 & 118 \\
\hline 1781 & 1.103 & 32 & 1881 & 1.046 & 93 & 1981 & 0.908 & 118 \\
\hline 1782 & 0.962 & 32 & 1882 & 1.184 & 93 & 1982 & 0.952 & 118 \\
\hline 1783 & 1.035 & 33 & 1883 & 1.163 & 93 & 1983 & 1.141 & 118 \\
\hline 1784 & 0.532 & 34 & 1884 & 1.144 & 93 & 1984 & 1.035 & 118 \\
\hline 1785 & 1.099 & 37 & 1885 & 1.095 & 94 & 1985 & 1.161 & 118 \\
\hline 1786 & 0.983 & 38 & 1886 & 1.085 & 94 & 1986 & 1.012 & 118 \\
\hline 1787 & 1.145 & 38 & 1887 & 1.183 & 94 & 1987 & 0.833 & 118 \\
\hline 1788 & 1.019 & 40 & 1888 & 1.066 & 94 & 1988 & 1.007 & 118 \\
\hline 1789 & 1.175 & 40 & 1889 & 1.085 & 94 & 1989 & 1.084 & 118 \\
\hline
\end{tabular}




\begin{tabular}{|c|c|c|c|c|c|c|c|c|}
\hline 1790 & 1.102 & 41 & 1890 & 0.977 & 94 & 1990 & 1.039 & 118 \\
\hline 1791 & 0.978 & 43 & 1891 & 1.068 & 94 & 1991 & 1.150 & 118 \\
\hline 1792 & 1.068 & 43 & 1892 & 0.909 & 94 & 1992 & 0.951 & 118 \\
\hline 1793 & 1.074 & 45 & 1893 & 1.157 & 96 & 1993 & 1.367 & 118 \\
\hline 1794 & 1.070 & 46 & 1894 & 1.213 & 97 & 1994 & 1.279 & 118 \\
\hline 1795 & 0.826 & 48 & 1895 & 1.123 & 97 & 1995 & 1.176 & 118 \\
\hline 1796 & 0.887 & 48 & 1896 & 1.049 & 97 & 1996 & 1.315 & 118 \\
\hline 1797 & 0.934 & 49 & 1897 & 1.325 & 97 & 1997 & 1.283 & 118 \\
\hline 1798 & 0.941 & 50 & 1898 & 1.216 & 98 & 1998 & 1.443 & 118 \\
\hline 1799 & 0.911 & 50 & 1899 & 1.314 & 98 & 1999 & 1.298 & 118 \\
\hline 1800 & 0.891 & 51 & 1900 & 1.182 & 100 & 2000 & 1.289 & 118 \\
\hline 1801 & 0.946 & 53 & 1901 & 1.018 & 101 & 2001 & 1.080 & 118 \\
\hline 1802 & 1.087 & 53 & 1902 & 1.118 & 103 & 2002 & 1.185 & 118 \\
\hline 1803 & 1.219 & 53 & 1903 & 1.129 & 103 & 2003 & 0.974 & 118 \\
\hline 1804 & 1.124 & 53 & 1904 & 1.018 & 103 & 2004 & 0.941 & 118 \\
\hline 1805 & 1.125 & 53 & 1905 & 1.004 & 104 & 2005 & 1.062 & 117 \\
\hline 1806 & 1.073 & 54 & 1906 & 1.075 & 104 & 2006 & 1.238 & 117 \\
\hline 1807 & 0.800 & 55 & 1907 & 0.831 & 104 & 2007 & 1.037 & 116 \\
\hline 1808 & 1.019 & 55 & 1908 & 0.836 & 104 & 2008 & 0.789 & 115 \\
\hline 1809 & 0.781 & 56 & 1909 & 0.695 & 104 & 2009 & 1.125 & 114 \\
\hline 1810 & 0.956 & 59 & 1910 & 0.811 & 107 & 2010 & 0.915 & 109 \\
\hline 1811 & 0.854 & 61 & 1911 & 0.680 & 107 & & & \\
\hline 1812 & 0.840 & 61 & 1912 & 0.899 & 108 & & & \\
\hline 1813 & 0.924 & 61 & 1913 & 0.792 & 108 & & & \\
\hline 1814 & 0.913 & 61 & 1914 & 0.877 & 108 & & & \\
\hline 1815 & 0.963 & 61 & 1915 & 0.635 & 108 & & & \\
\hline 1816 & 0.902 & 62 & 1916 & 0.696 & 108 & & & \\
\hline 1817 & 0.868 & 63 & 1917 & 0.651 & 108 & & & \\
\hline 1818 & 0.582 & 64 & 1918 & 0.619 & 108 & & & \\
\hline 1819 & 0.946 & 64 & 1919 & 0.621 & 108 & & & \\
\hline 1820 & 1.044 & 65 & 1920 & 0.759 & 108 & & & \\
\hline 1821 & 0.881 & 65 & 1921 & 0.769 & 108 & & & \\
\hline 1822 & 1.088 & 65 & 1922 & 0.740 & 108 & & & \\
\hline 1823 & 0.924 & 67 & 1923 & 0.934 & 108 & & & \\
\hline 1824 & 0.896 & 67 & 1924 & 0.850 & 108 & & & \\
\hline
\end{tabular}




\section{Supplementary Tables and Figures}

Table S1 Information of the weather stations and gridded data nearest to sampling sites.

\begin{tabular}{llllll}
\hline Site & Latitude $(\mathrm{N})$ & Longitude $(\mathrm{E})$ & Altitude $(\mathrm{m})$ & Time-Span & Resolution \\
\hline Xiaoergou & $49^{\circ} 12^{\prime}$ & $123^{\circ} 43^{\prime}$ & 286.1 & $1957-2014$ & - \\
TS3.23 Mean T & $51.25^{\circ}$ & $123.75^{\circ}$ & - & $1901-2014$ & $0.5^{\circ} * 0.5^{\circ}$ \\
GPCC Precipitation & $51.25^{\circ}$ & $123.75^{\circ}$ & - & $1901-2014$ & $2.5^{\circ} * 2.5^{\circ}$ \\
Dai-PDSI & $51.25^{\circ}$ & $123.75^{\circ}$ & - & $1911-2013$ & $2.5^{\circ} * 2.5^{\circ}$ \\
Cook-PDSI & $51.25^{\circ}$ & $123.75^{\circ}$ & - & $1725-2005$ & $2.5^{\circ} * 2.5^{\circ}$ \\
sc-PDSI & $51.25^{\circ}$ & $123.75^{\circ}$ & - & $1901-2013$ & $0.5^{\circ} * 0.5^{\circ}$ \\
CSIC-SPEI & $51.25^{\circ}$ & $123.75^{\circ}$ & - & $1901-2012$ & $0.5^{\circ} * 0.5^{\circ}$ \\
\hline
\end{tabular}




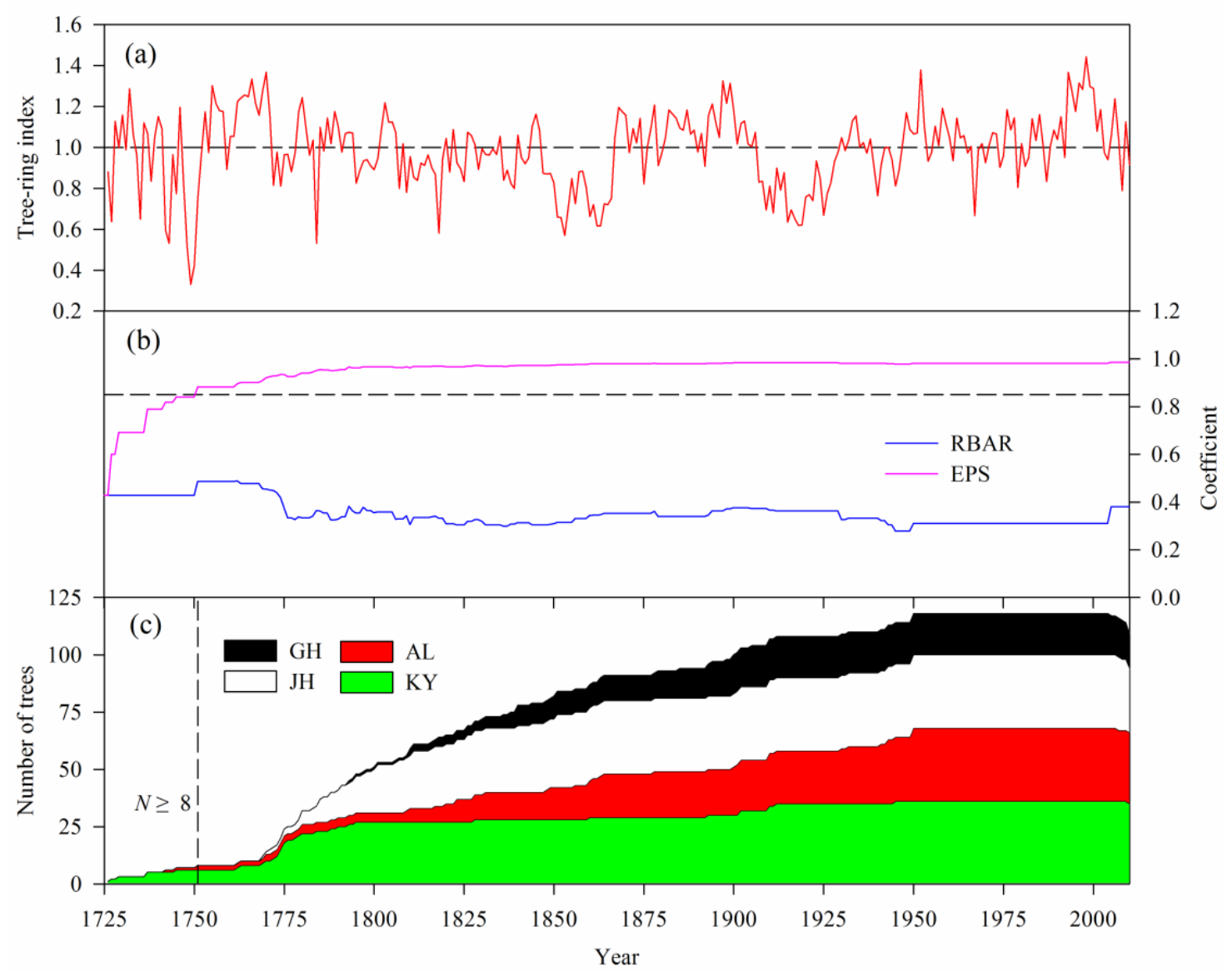

Fig. S1 The regional tree-ring width chronology of Pinus sylvestris var. mongolica in the Daxing'an

Mountains (a). The RBAR, EPS (b), and sample size (c) of the chronology are also shown. RBAR and EPS are computed using 51-year windows. The reliable segment of the chronology is determined by the EPS value $>0.85$. 
(a)

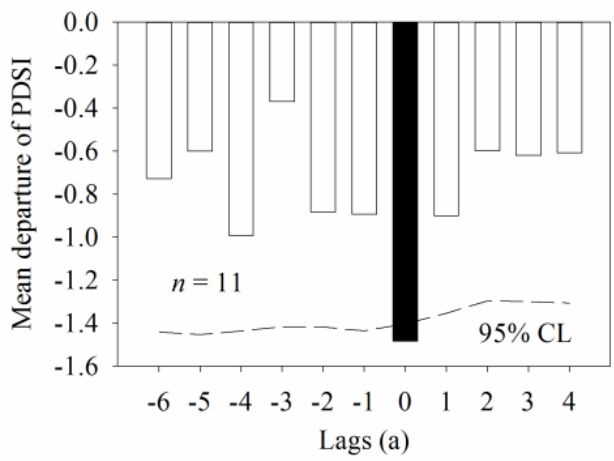

(b)

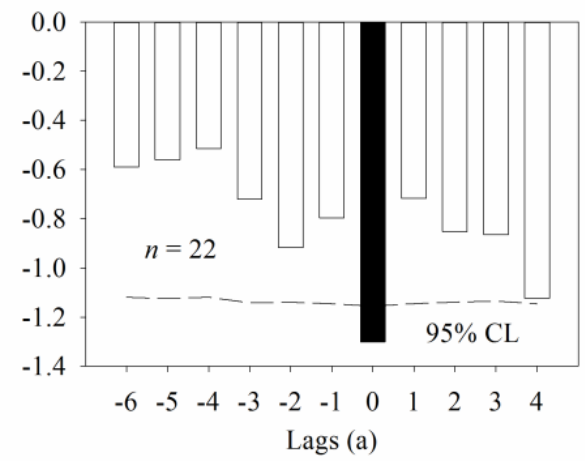

Fig. S2 Superposed epoch analyses of reconstructed Dai-PDSI (1751-2010) with regional wildfire years of (a) Mengkeshan (1743-2010) and (b) Pangu (1767-2010) in the Daxing'an Mountains, northeast China. The analysis window includes six and four years before and after fire year (year "0"). Black bars indicate significant departures $(p<0.05$; dashed lines) from mean conditions. 


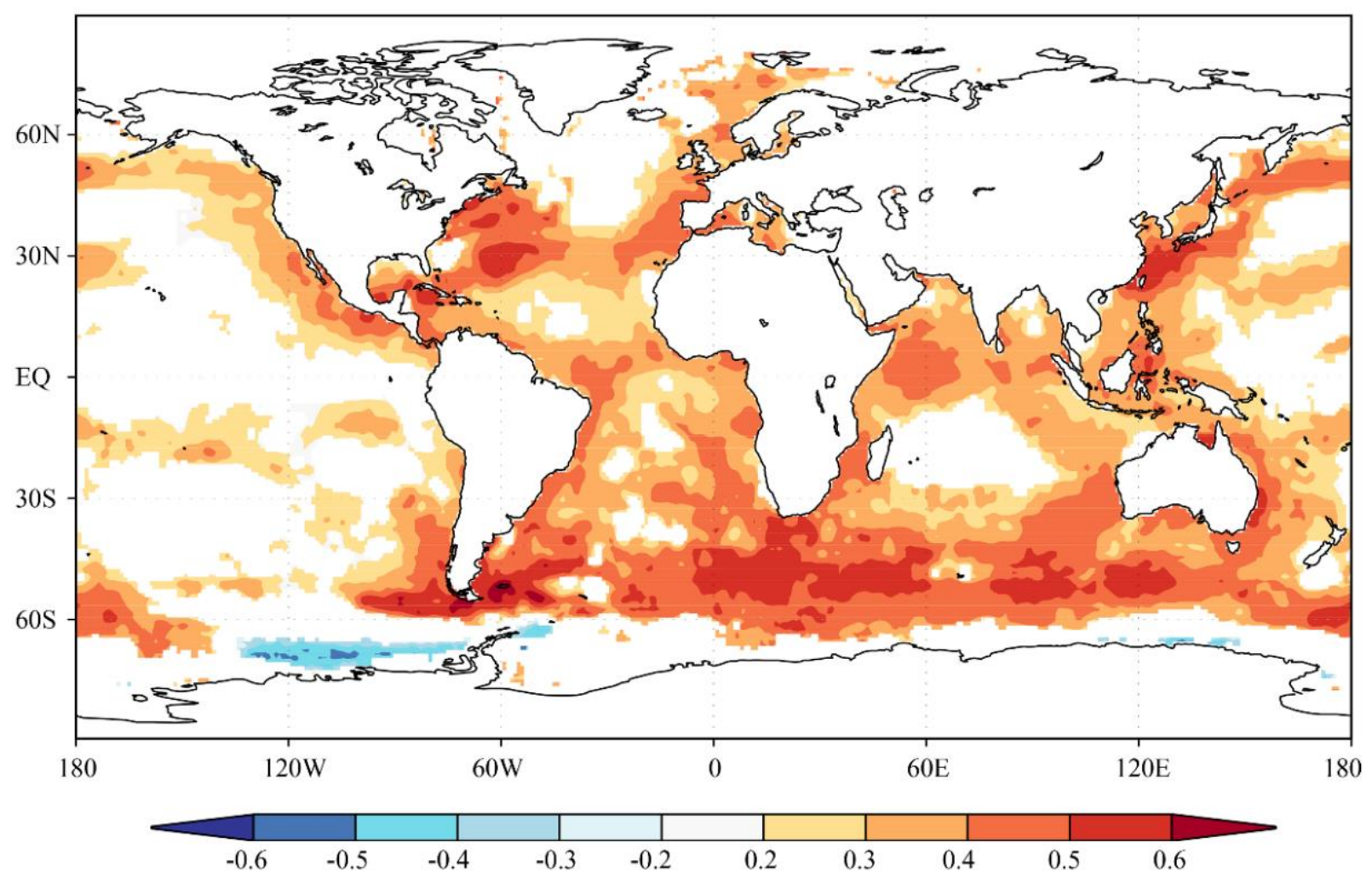

Fig. S3 Spatial correlations between the reconstructed PDSI and sea surface temperature in global scale.

The spatial correlation covered a time span from AD 1911 to 2010. 
(a)

NCEP/NCAR Reanalysis

Surface Potential Evoporation (W/m^2) Composite Anomaly 1981-2010 climo

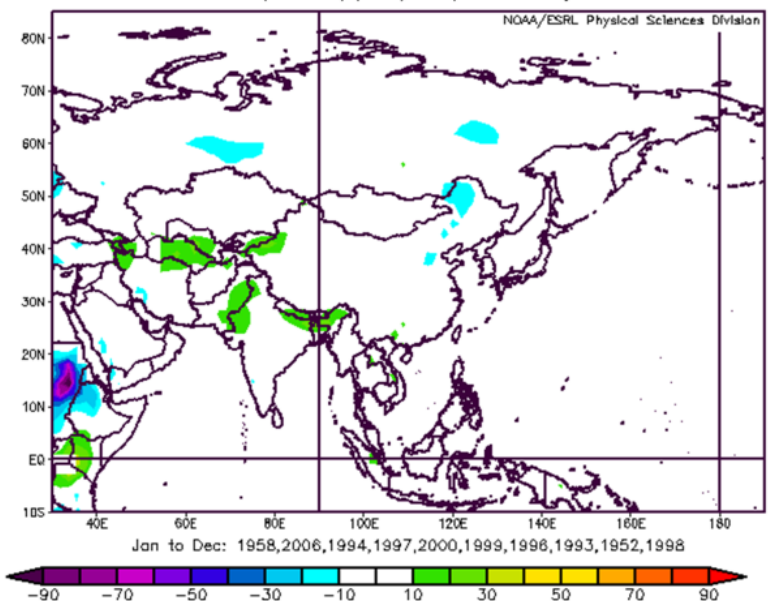

(b)

NCEP/NCAR Reonalysis

Surface Potential Evoporation (W/m^2) Composite Anomaly 1981-2010 climo

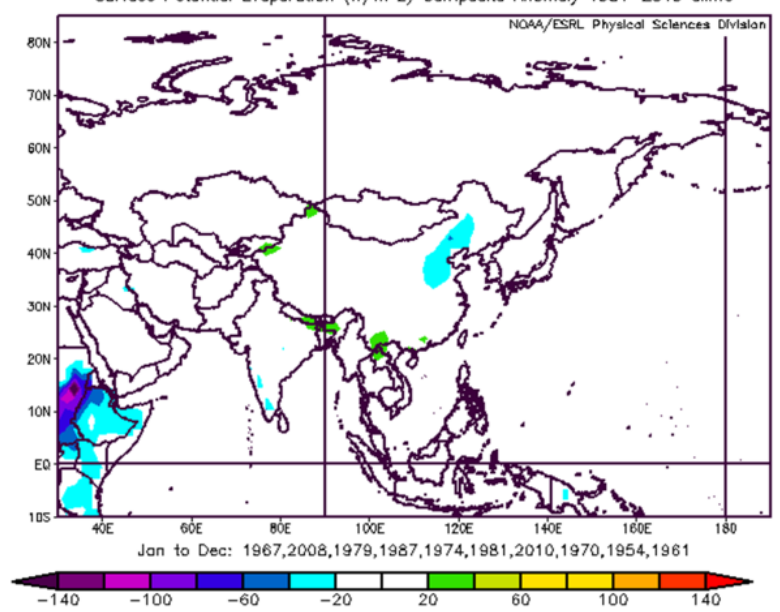

Fig. S4 Composite anomaly maps of the surface potential evaporation $\left(\mathrm{W} / \mathrm{m}^{2}\right)$ (from January to

December) for the 10 wettest (a) and driest (b) years of the reconstruction Dai-PDSI during the period 1948-2010. 